\title{
Estudio de la Variabilidad de Proceso en el Área de Envasado de un Producto en Polvo
}

\author{
Elizabeth E. Díaz, Carlos Díaz, Luis C. Flores y Santiago Heyser \\ Instituto Tecnológico de Orizaba, Departamento de Estudios de Postgrado e Investigación, \\ Av. Oriente 9 No. 852, 94329 Orizaba, Veracruz-México \\ (e-mail: Iyszy_dc@hotmail.com, carlosdiazramos@yahoo.com.mx ,Icfloresa@yaho.com, \\ sheyserf@yahoo.com.mx)
}

\begin{abstract}
Resumen
Se presenta los resultados de un estudio sobre la optimización de la cantidad de producto en polvo depositada en bolsas, en el área de envasado de una industria que se dedica al procesamiento de productos en grano y en polvo. Se aplicaron técnicas de control estadístico de procesos como: cartas de control y análisis de la capacidad de proceso. Además, se utilizó un diseño factorial $2^{5}$ para obtener las condiciones óptimas de operación del proceso de envasado. La aplicación de dichas herramientas estadísticas permitió cumplir con las especificaciones de las normas mexicanas de calidad, obteniéndose una reducción del 95\% en las pérdidas de producto terminado. Basándose en los resultados se concluye que la aplicación de técnicas y herramientas estadísticas, son de gran utilidad para mejorar el control de productos y procesos.
\end{abstract}

Palabras clave: variabilidad, control estadístico de procesos, cartas de control, diseños factoriales,

\section{A Study on Process Variability in the Packing Area of a Powder Product}

\begin{abstract}
The results of a study on the optimization of the amount of powder product deposited in bags, in the packaging area of an industry that is dedicated to the processing of grain and powder products are presented. Statistical process control techniques such as control charts and process capability analysis were applied. Also, a factorial design $2^{5}$ for obtaining the optimal operating conditions was used. The application of these statistical tools allowed fulfilling the specifications of the Mexican standards, obtaining a 95\% reduction in the loss of finished product. Based on the results, it is concluded that the application of statistical tools and techniques, are very useful to improve product and process control.
\end{abstract}

Keywords: variability, capability of process, control charts, factorial design, statistical process control 


\section{INTRODUCCIÓN}

La competitividad de una empresa y la satisfacción del cliente están determinadas por la calidad del producto, el precio y la calidad del servicio. Se es más competitivo si se puede ofrecer mejor calidad, a bajo precio y en menor tiempo. Respecto a la calidad existen varias definiciones, una que sintetiza la idea de enfocar la empresa hacia los clientes es (Montgomery, 2001): "La Calidad es inversamente proporcional a la variabilidad en un proceso". En un proceso industrial interactúan materiales, máquinas, mano de obra (gente), mediciones, medio ambiente y métodos (6 $\left.\mathrm{M}^{\prime} \mathrm{s}\right)$. Estos seis elementos determinan de manera global todo proceso y cada uno aporta algo de la variabilidad ( $\mathrm{y}$ a la calidad) de la salida del proceso (Evans y William, 2005).

Con el transcurso del tiempo ocurren cambios frecuentes en estas $6 \mathrm{M}$ 's, por lo que las grandes empresas mundiales han dedicado gran importancia en la necesidad de monitorear de manera constante los signos vitales del proceso, lo que se conoce como: características o indicadores críticos para la calidad ó CTQ's (Lloyd, 2003). Para poder monitorearlos o medirlos se debe hacer uso de técnicas estadísticas, desde la aplicación de estadística descriptiva, hasta el control estadístico de procesos (SPC), incluyendo técnicas avanzadas, inclusive técnicas multivariadas (Peña y Prat, 1986). El objetivo del SPC es el de establecer un sistema de observación, permanente e inteligente, que detecte precozmente la aparición de causas especiales de variabilidad y ayude a identificar su origen, con el fin último de eliminarlas del proceso y de tomar medidas que eviten su reaparición en el futuro.

Casi todas las técnicas del Control Estadístico de Procesos que se utilizan hoy en día, fueron desarrolladas durante el siglo pasado. El trabajo pionero llevado a cabo por el Dr. Walter Shewhart y su equipo de colaboradores en 1924 en los Laboratorios Bell, sentaron las bases del SPC moderno, a tal grado, que hoy en día se sigue publicando su libro "Economic Control of Quality of Manufactured Product", por la American Society for Quality (ASQ).

Nadie ha tenido tanto impacto en los principios estadísticos del diseño de experimentos en su tiempo como Ronald A. Fisher. En octubre de 1919, Fisher fue contratado en Rothamsted Experimental Station, cerca de Harpenden, Inglaterra. Le pidieron que trabajara con ellos de seis meses a un año, para aplicar un exhaustivo análisis estadístico a los datos de investigaciones agrícolas que el personal había recolectado. Fue durante su ejercicio en Rothamsted, donde permaneció hasta 1933, que desarrolló y consolidó los principios básicos de diseño y análisis que hasta la fecha son prácticas necesarias para llegar a resultados de investigación válidos. De 1919 a 1925 estudió y analizó experimentos relativos al trigo que se habían realizado desde 1843. De sus investigaciones estadísticas de éstos y otros experimentos, Fisher desarrolló el análisis de varianza y unificó sus ideas básicas sobre los principios del diseño de experimentos.

Al finalizar la Segunda Guerra Mundial se incrementó el interés en la calidad, primordialmente en las industrias japonesas, quienes estuvieron ayudadas por el Dr. W. E. Deming. Desde los años 1980's, las industrias de Estados Unidos empezaron a implementar la calidad en todos sus productos. Estas estuvieron asesoradas por el Dr. Genichi Taguchi, Philip Crosby, Dr. Deming, y el Dr. Joseph M. Juran. Las industrias en los años 1980's también se vieron beneficiadas por las contribuciones del Dr. Taguchi acerca del Diseño de Experimentos, Función de pérdida y Diseños Robustos.

En la actualidad, son muchos los trabajos reportados tanto en lo que se relaciona a desarrollos teóricos, como a aplicaciones del SPC. Otros autores, hacen una revisión bibliográfica acerca de las propiedades de las cartas de control; Woodall (2006), reporta el uso de las cartas de control en el área de la salud; Bulba y Ho (2006), proponen un intervalo de confianza para índices de capacidad de proceso de variables no observables; Denniston (2006), por su parte, al analizar los índices de capacidad de proceso, motiva para el uso de Cpm; Han (2006), por otro lado, reportó un estudio sobre el efecto de las pruebas de normalidad sobre la estimación de los índices de capacidad de proceso; Bisgaard (2008), realizó un estudio acerca del SPC y el diseño de experimentos; Au y Tsui (2007), aplicaron el SPC al monitoreo de actividades económicas; Shore (2006), utiliza de manera original las Cartas de Control en la Teoría de Colas; Albers y Kallenberg (2006), presentan un tipo de cartas autoajustables; Koning (2006), propone un método general para construir cartas de control 
para observaciones individuales; y Tsai (2007), trata sobre el diseño de cartas de control para medias, en casos en donde la distribución normal es sesgada.

Lo antes expuesto, pone de manifiesto la gran utilidad práctica que tienen todas estas técnicas en la solución de problemas reales en la industria.

\section{METODOLOGÍA}

\section{Planteamiento del problema}

En este trabajo se describe la aplicación de técnicas de Control Estadístico de Proceso y de Diseño de Experimentos, a una industria procesadora de producto en grano y en polvo. Específicamente la investigación aplicada se llevó a cabo en el departamento de envasado; este proceso para efectos del presente estudio, inicia en la "tolva dosificadora" cuyo contenido proviene de etapas anteriores del proceso a través de una banda vibradora que transporta el producto en polvo. Esta tolva se encuentra integrada a la máquina de envasado ENV-A-FLEX, lugar en donde se lleva a cabo la etapa final del llenado de las bolsas. El producto de la tolva dosificadora pasa directamente por gravedad a cada uno de 6 vasos con que cuenta la máquina, los cuales giran en torno a la tolva dosificadora a determinada velocidad. La velocidad a la cual giran los vasos es controlada por medio de un mecanismo de acción manual.

Posteriormente, el producto es descargado a las bolsas mediante el vaciado de los vasos que se encuentran en movimiento sincronizado. Una vez que se llena cada bolsa de producto, inmediatamente es sellada mediante unas placas metálicas que operan a cierta temperatura (cierre térmico). Enseguida, la bolsa llena es separada de la bobina mediante la acción de un mecanismo de corte; así, la bolsa cae directamente a una banda transportadora, para ser trasladada directamente al área de empacado y fleje. Mientras esto sucede, el producto envasado es sometido a un proceso de inspección aleatorio, que consiste en determinar el peso real de producto contenido en cada bolsa y contrastar dicho peso con el establecido en las normas. Es preciso comentar que en esta etapa del proceso se detectaba una cantidad considerable de producto no conforme; además, que este hecho obligaba a revisar muy frecuentemente la calibración de los vasos, los cuales tenían que ajustarse para supuestamente trasladar la media del proceso lo más cerca que fuera posible a su valor nominal.

La empresa, por políticas propias, se compromete a cumplir con dos tipos de normas; una obligatoria y la otra de carácter voluntario. El producto final es envasado en 4 diferentes presentaciones: 25, 100, 250 y 500 gramos respectivamente; la tolerancia máxima permitida es de $\pm 1 \%$. El estudio se llevó a cabo con la presentación de 25 gramos, cuya tolerancia es de \pm 0.25 gramos. Actualmente, el contenido de producto de este tipo de bolsas tiene una variación de \pm 11 gramos con respecto a su valor nominal, variabilidad que a todas luces es inaceptable, ya que representa un $\pm 44 \%$ con respecto a la media deseada. Lógicamente esto tiene un impacto sumamente negativo sobre los costos de producción, debido a las pérdidas de producto terminado; además de impactar negativamente en el grado de satisfacción del cliente.

La metodología aplicada consta de 6 etapas a saber: experimentación preliminar, análisis de resultados, diseño del experimento, implementación, análisis e interpretación de resultados y corrida de confirmación (Gijo, 2005). Los beneficios de la aplicación de esta metodología consistieron en la identificación de las causas de la variabilidad, la centralización del proceso y la disminución significativa la variación, dando por resultado una reducción del 95\% en las pérdidas de producto terminado con las consecuentes ventajas económicas.

\section{Medición de la variación de proceso mediante el uso de Cartas de control}

El objetivo básico de un gráfico de control es, observar y analizar con datos estadísticos, la variabilidad y el comportamiento de un proceso a través del tiempo, para una o varias características de calidad o variables de salida. Por tanto, primeramente se utilizaron estas herramientas para valorar el comportamiento del proceso. Se aplicaron gráficos de control de medias y rangos tomando muestras de 5 observaciones por cada vaso, para hacer un total de 30 observaciones (6 vasos). Las 
muestras fueron tomadas a intervalos de 20 minutos a lo largo del proceso de producción. Los gráficos resultantes se dan en la figura 1.

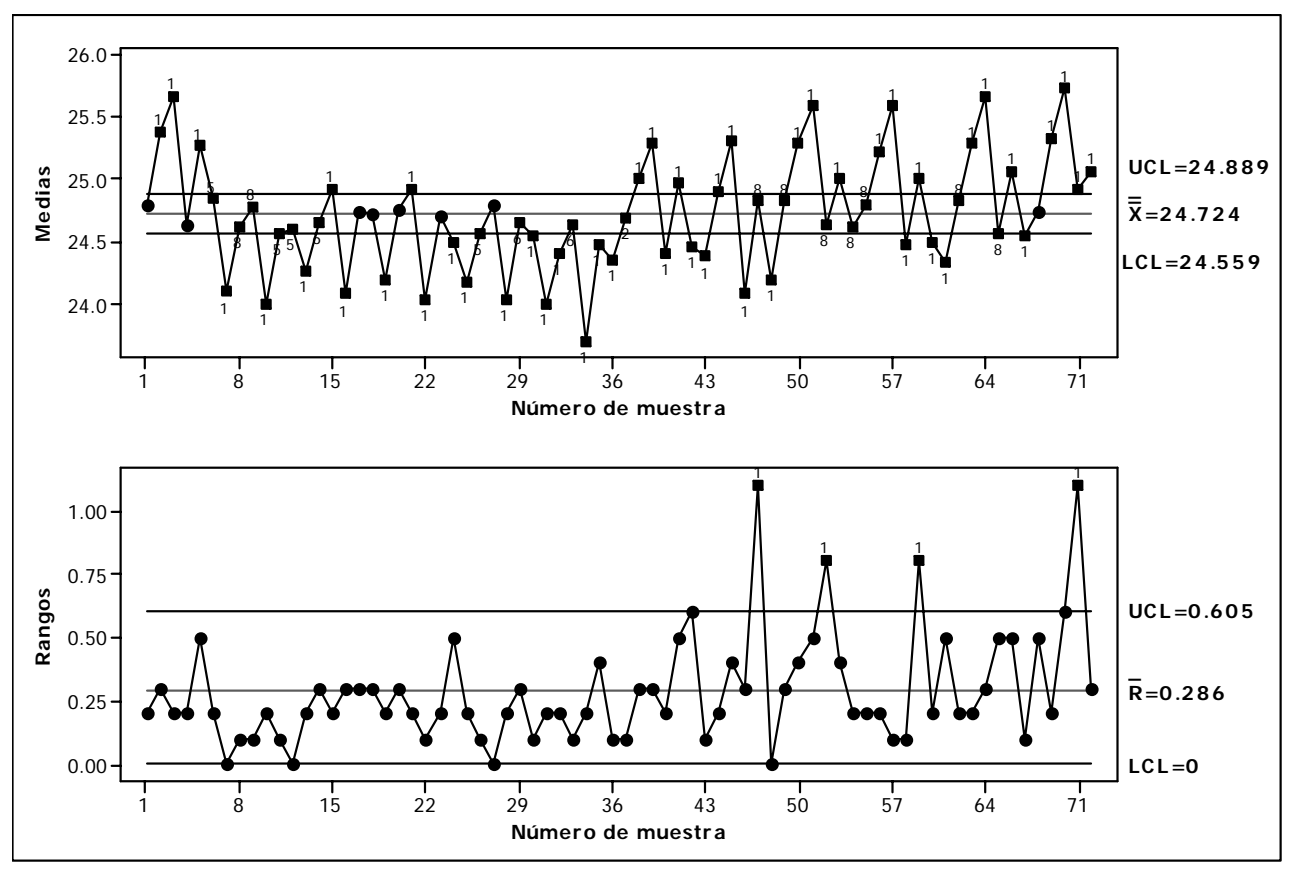

Fig. 1: Cartas de control de medias y de rangos para la máquina ENV-A-FLEX.

La carta de control de medias, deja muy claro que el proceso no se encuentra bajo control estadístico, debido a que la mayoría de los puntos caen fuera de los límites de control. Además, se observan patrones de comportamiento bien definidos que indican cambios en la media durante ciertos periodos de tiempo, en algunos casos por encima de la línea central, y en otros por debajo. Estos comportamientos anómalos señalan la presencia de causas especiales de variación. Dichos desplazamientos o cambios en el nivel del proceso se deben a la introducción de nuevos trabajadores, materiales o métodos, cambios en los métodos de inspección, una mayor o menor atención de los trabajadores.

Por otro lado, la gráfica de rangos, muestra dos puntos por encima del límite superior de control, indicando con ello la presencia de causas asignables de variación. Una investigación adicional arrojó que esto se debe a variables tales como temperatura, fatiga o cansancio del operario, rotación regular de operarios o máquinas, fluctuación de voltaje o presión, o de alguna otra variable en el equipo de producción.

\section{Diseño del experimento}

Dada la gran variabilidad detectada en las cartas de control, se optó por utilizar experimentos diseñados (DOE) para mejorar el desempeño del proceso y tener una base sólida para evaluar su capacidad. Los experimentos diseñados son una serie de pruebas en las cuales se inducen cambios deliberados en las variables de entrada o variables independientes de un proceso o sistema, de manera que sea posible observar e identificar las causas de los cambios en la respuesta de salida, esto se realiza con la finalidad de identificar qué variables o factores tienen mayor influencia en la respuesta observada, determinar el mejor nivel de los factores o variables y llevar la respuesta a su nivel óptimo; ya sea que se trate de una característica de calidad del tipo menor-mejor, mayor-mejor o nominal-mejor. Esto, a la luz de minimizar los efectos de aquellas variables o factores incontrolables en el proceso. Este enfoque se utiliza, lo mismo en cualquier rama de investigación científica, ya sea, ingeniería, biología, medicina, economía, sociología, psicología, marketing, agricultura, etc. En las etapas iniciales de la planeación y diseño de un experimento, es recomendable utilizar técnicas como la lluvia de ideas, las cuales, no por ser simples dejan de ser poderosas para motivar el pensamiento creativo y detectar ideas innovadoras relacionadas con un problema en particular. El equipo de trabajo formado para la investigación estuvo integrado por los 
operarios y los jefes del área de envasado de la planta. Las actividades desarrolladas en las diferentes sesiones (lluvia de ideas) fueron las siguientes:

- $\quad$ Presentación del proyecto y definición del problema.

- $\quad$ Elección del coordinador general del proyecto.

- $\quad$ Asignación de tareas a cada miembro del equipo.

- $\quad$ Elaboración de un reporte individual.

- $\quad$ Sesión de lluvia de ideas para la identificación de las causas responsables del problema.

- $\quad$ Elaboración de un Diagrama de Causa-Efecto.

- $\quad$ Revisión del Diagrama de Causa-Efecto elaborado.

- $\quad$ Selección de las causas más importantes.

El diagrama de causa-efecto resultante se muestra en la figura 2. En este diagrama, las causas se encuentran agrupadas de acuerdo a las categorías: métodos de trabajo, mano de obra, materiales, máquinas, medición y medio ambiente.

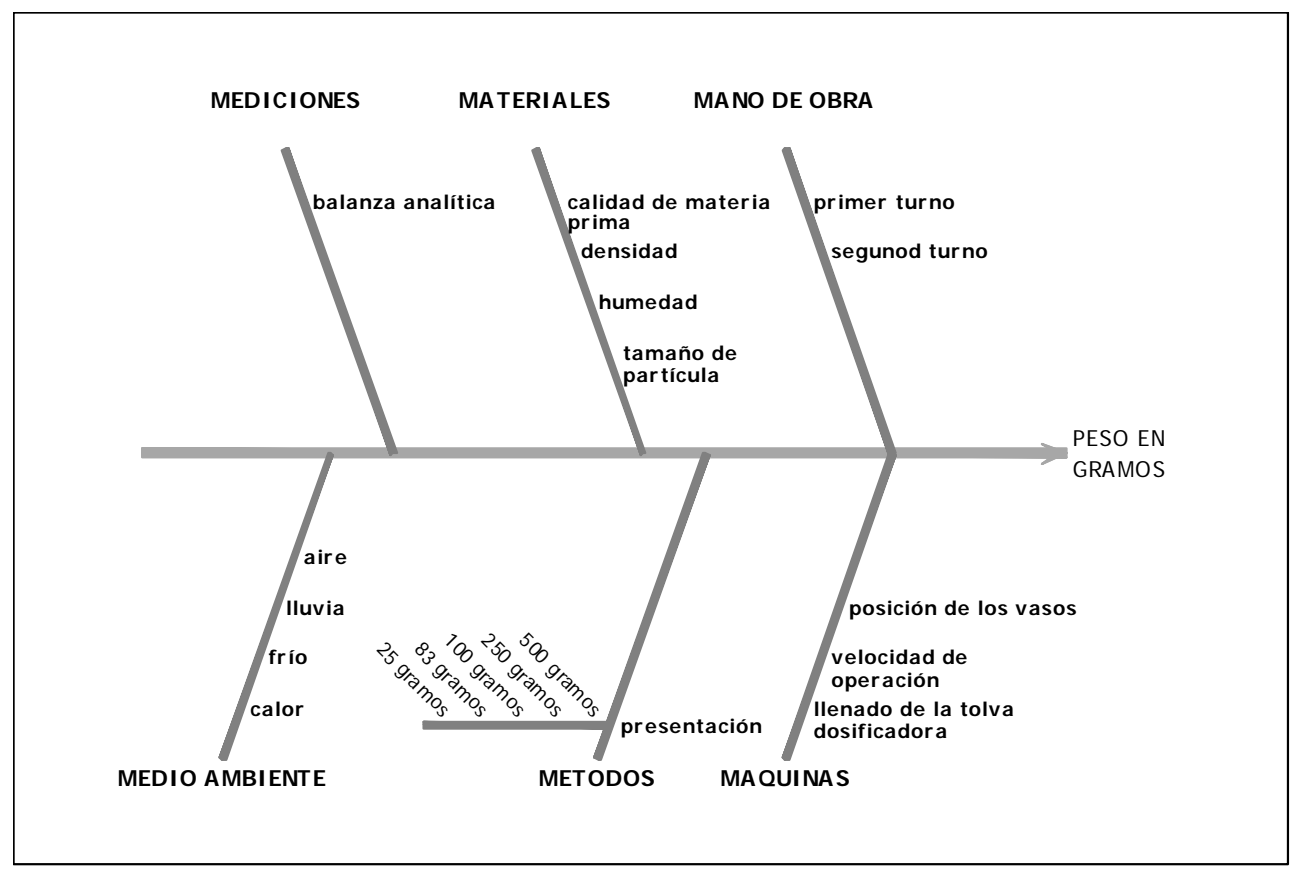

Fig. 2: Diagrama de Causa-Efecto.

Tabla 1; Niveles de los factores

\begin{tabular}{|l|l|l|}
\hline FACTOR & NIVEL BAJO (-1) & NIVEL ALTO (1) \\
\hline $\begin{array}{l}\text { A=llenado de la tolva } \\
\text { dosificadora }\end{array}$ & $\begin{array}{l}\text { mínima cantidad posible de } \\
\text { producto }\end{array}$ & $\begin{array}{l}\text { máxima cantidad posible de } \\
\text { producto }\end{array}$ \\
\hline $\begin{array}{l}\text { B=velocidad de operación de } \\
\text { la máquina }\end{array}$ & 20 bolsas/min & 35 bolsas/min \\
\hline C=calibración de los vasos & completamente cerrados & completamente abiertos \\
\hline D=posición de los vasos & posición \#1 & posición \# 6 \\
\hline E=densidad & $326 \mathrm{~g} / \mathrm{l}$. humedad 1.52\% & $327 \mathrm{~g} / \mathrm{l}$. humedad $2.60 \%$ \\
\hline
\end{tabular}

Una vez construido y analizado el diagrama causa-efecto, y tomando en cuenta que; la tolva dosificadora cambia su nivel con cierta frecuencia, la velocidad de operación se controla manualmente y por tanto puede constituir una fuente de variación importante, la calibración de los vasos también se lleva a cabo de forma manual, los vasos pueden ocupar diferentes posiciones en la máquina y se sospecha que esto puede influir en la variación de la característica de calidad, la humedad y el tamaño de la partícula son los factores que aparentemente más influyen en la variabilidad de la respuesta; se decidió incluir en la experimentación a cinco factores, a saber: 
llenado de la tova dosificadora (A), velocidad de operación (B), calibración de vasos (C), posición de vasos (D) y densidad del producto (E). Por tratarse de un primer experimento, se optó por utilizar un diseño $2^{5}$ (Mathews, 2005). En la tabla 1 se identifican los niveles de cada factor.

\section{RESULTADOS Y DISCUSIÓN}

Se llevó a cabo el experimento con seis réplicas en cada una de las 32 corridas experimentales aplicadas en forma aleatoria, los resultados se analizaron utilizando MINITAB versión 15 (figura 3).

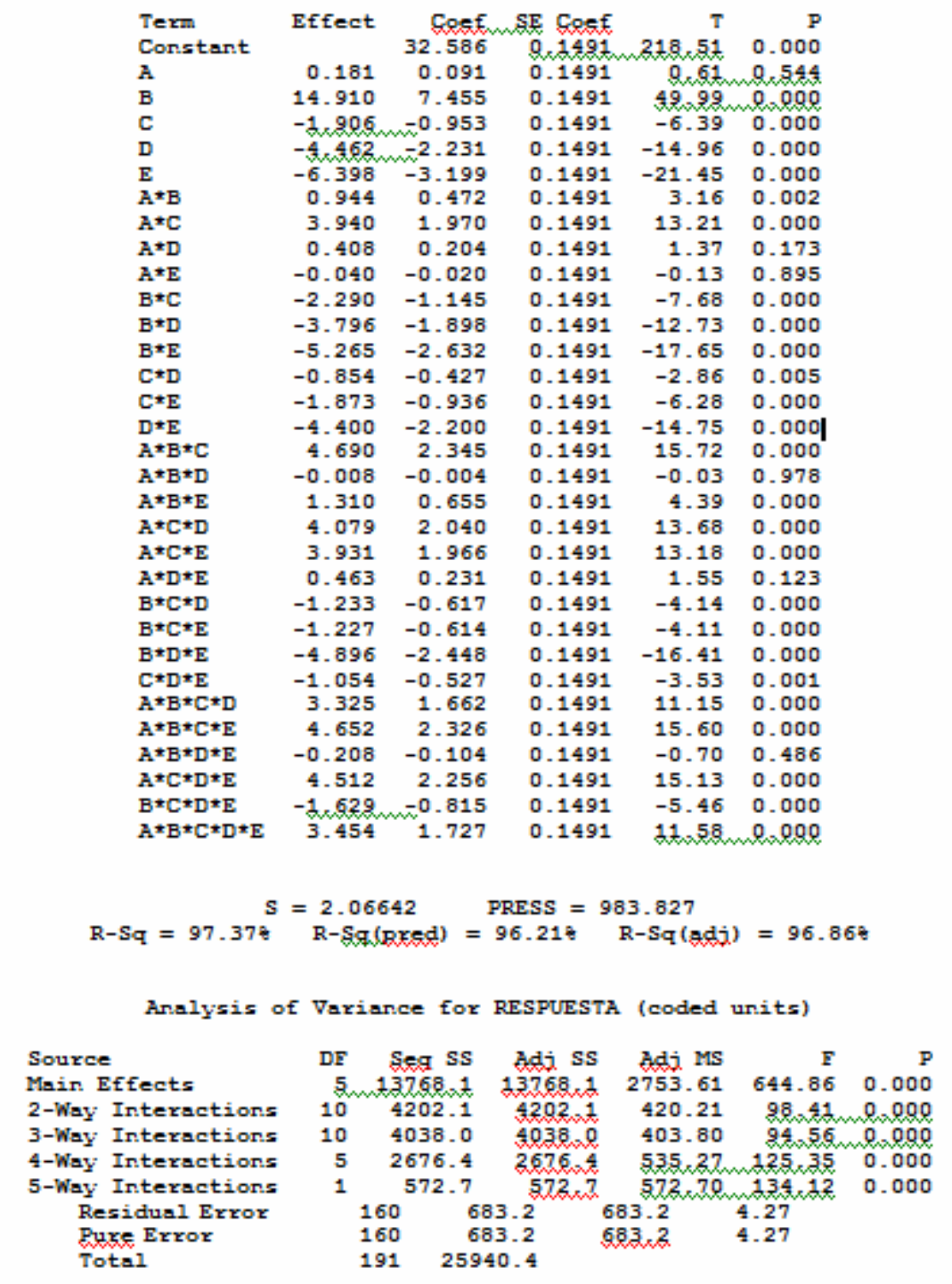

Fig. 3; Resultados del ANOVA con MINITAB

Del análisis de varianza (ANOVA), se observa que todos los factores e interacciones son significativos a excepción de: $A, A D, A E, A B D, A D E, A B D E$. La figura 4 muestra los diagramas de efectos principales del experimento en donde se puede apreciar objetivamente la significancia de los factores principales

Analizados todos los resultados provenientes del diseño se pueden identificar los niveles de los factores significativos que pueden desplazar al proceso hacia el valor nominal deseado (25 gramos), estos niveles se muestran en la tabla 2. Bajo estas condiciones de operación del proceso, se espera que la media del peso del producto sea de 25.033 gramos, con lo que se puede considerar que la característica de calidad bajo estudio se puede centrar en su valor nominal. Se llevó a cabo una corrida experimental de confirmación con la finalidad de validar empíricamente los resultados del experimento. Los resultados obtenidos dieron una respuesta promedio de 24.933 gramos, con estimado a priori de la desviación estándar de 0.137 gramos, con lo que se confirma que aplicando 
las nuevas condiciones operativas del proceso, se logra cumplir con el valor nominal y disminuir la variación drásticamente. Una prueba adicional de hipótesis, nos lleva a suponer que el peso promedio depositado en las bolsas es realmente de 25 gramos.

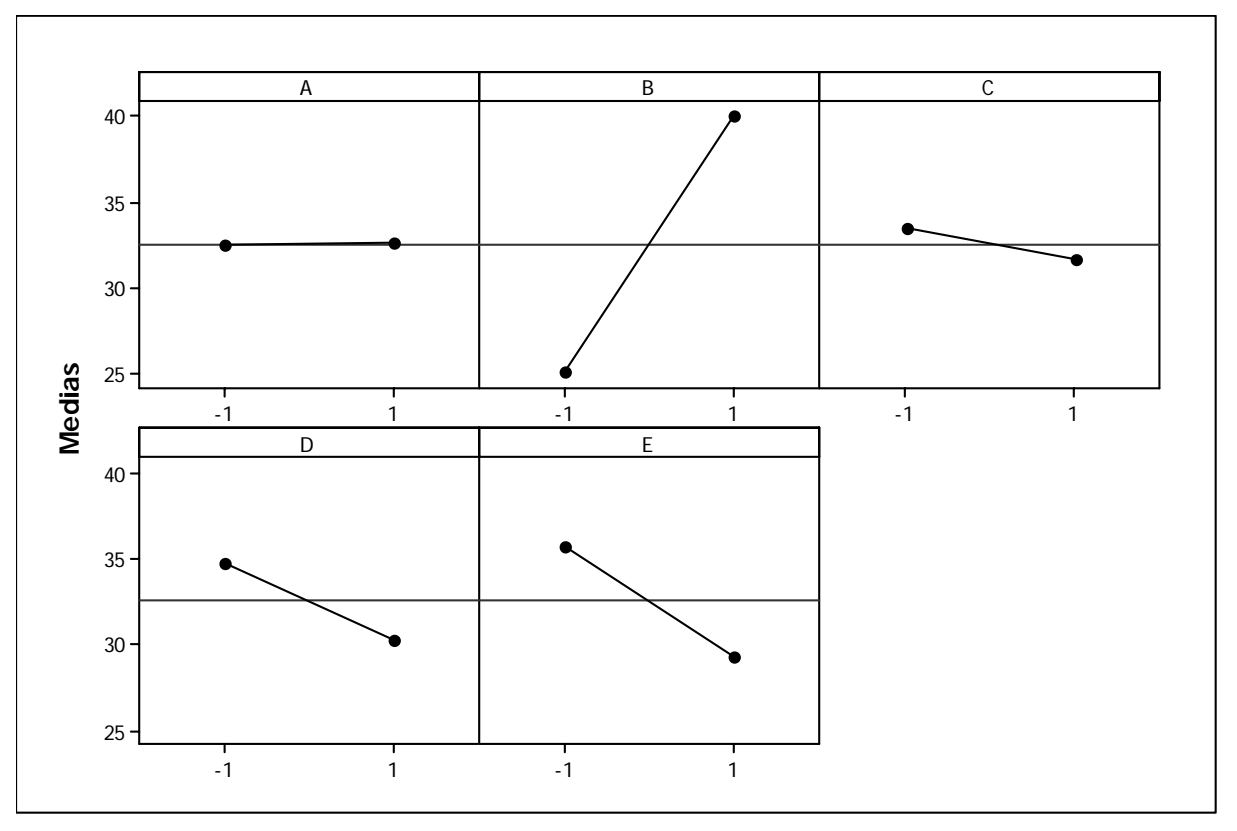

Fig. 4; Diagrama de efectos principales

Tabla 2: Niveles óptimos para cada factor

\begin{tabular}{|l|l|}
\hline \multicolumn{1}{|c|}{ Factor } & \multicolumn{1}{c|}{ Nivel } \\
\hline A, llenado de la tolva dosificadora & Máxima cantidad posible de café \\
\hline B, velocidad de operación & 20 bolsas/minuto \\
\hline C, calibración de los vasos & Completamente cerrados \\
\hline D, posición de los vasos & Posición \# 1 \\
\hline E, densidad del producto & $326 \mathrm{~g} /$. humedad $1.52 \%$ \\
\hline
\end{tabular}

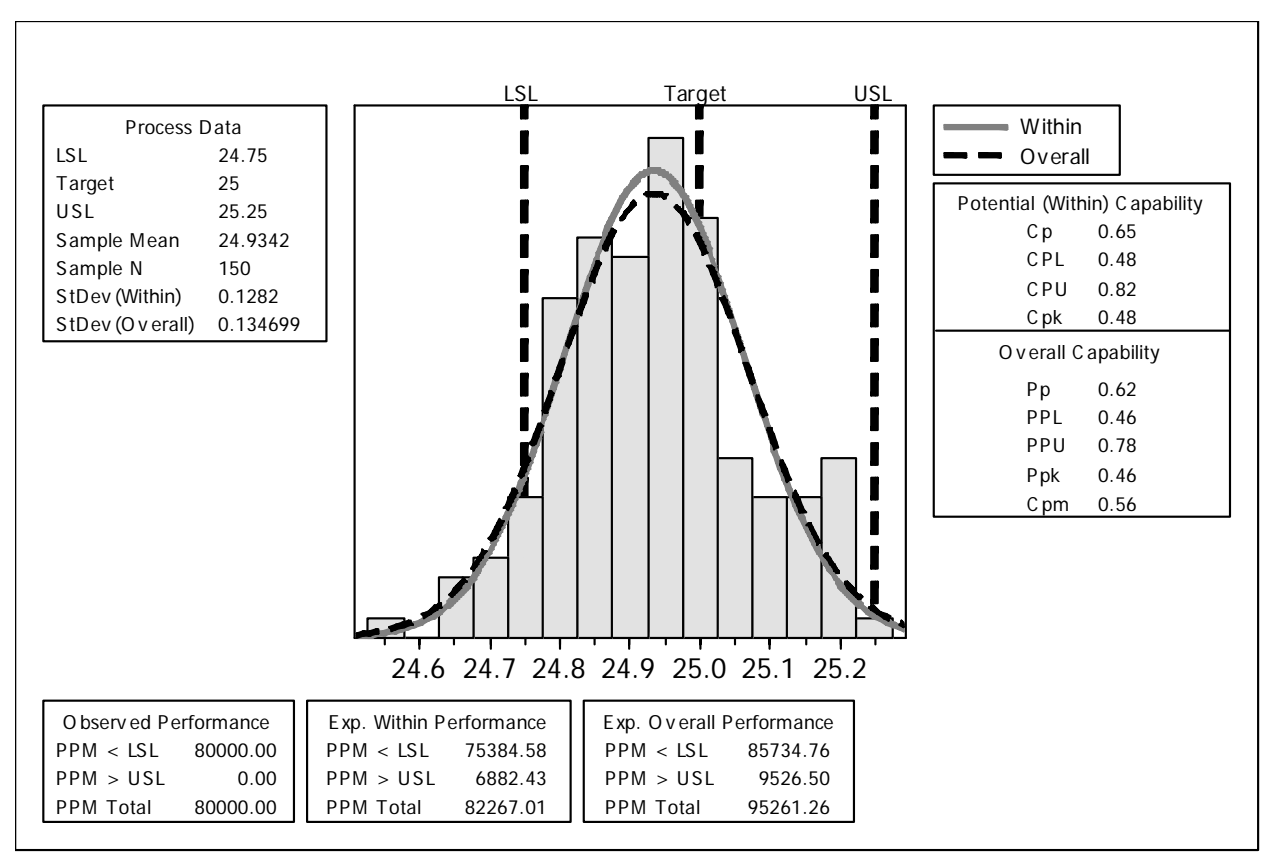

Fig. 5; Capacidad de proceso calculado para HRTE5E después del experimento 
Con los datos obtenidos de la corrida experimental de confirmación, se realizó un gráfico de control de medias del cual se concluye que el proceso se encuentra bajo control estadístico (Kotz y Johnson, 2002). Una prueba de bondad de ajuste aplicada a los datos, confirmó la presencia de normalidad en los mismos (Han, 2006), gracias a lo cual es posible realizar el estudio de capacidad de proceso (Gutiérrez y De la Vara, 2004; Polansky, 2005). La Figura 5 muestra los resultados del análisis de capacidad de proceso obteniéndose un $C_{p}$ equivalente a 0.65 , lo cual indica que el proceso aún no es capaz, y gran parte de esto se debe a la variabilidad aún presentada en el proceso (Denniston, 2006; Hung-Ching y Sheen-Gwo, 2005), y aún existe cantidad de producto considerable que está por debajo del límite de especificación inferior.

\section{CONCLUSIONES}

El uso adecuado de técnicas del SPC y de diseño de experimentos trae consigo grandes beneficios en los procesos de producción de bienes y/o servicios. Los resultados reportados en este trabajo demuestran como fue posible, en un principio, detectar la enorme variación que el proceso de envasado tenía, mediante el uso de las cartas de control; y después, reducir significativamente la variación y centrar el proceso a su valor nominal, a través de la implementación un experimento factorial $2^{5}$.

El impacto del proyecto de investigación se reflejó inmediatamente tanto en la reducción de las pérdidas de producto, así como en lo que más importa a la empresa, los costos. El efecto del antes y el después se presenta en la tabla 3.

Tabla 3; Ahorro esperado con las nuevas condiciones de operación del proceso

\begin{tabular}{|c|c|c|}
\hline & ANTES & DESPUÉS \\
\hline $\begin{array}{c}\text { Pérdida de producto por } \\
\text { bolsa producida }\end{array}$ & 0.70 gramos & 0.033 gramos \\
\hline Pérdida económica al mes & 201.92 US & 9.37 US \\
\hline Pérdida económica al año & $2,423.07$ US & 112.5 US \\
\hline
\end{tabular}

Este estudio se llevó a cabo para la presentación de 25 gramos, los motivos por los cuales se hizo esto son los siguientes; primero, porque esta era la presentación que se estaba envasando al momento de realizar el estudio; segundo, debido a que este tipo de producto solo representa el $4 \%$ de la producción total del departamento de envasado; tercero, el de mayor importancia, por el escepticismo que todavía impera en muchas empresas en México, respecto al beneficio que tiene la aplicación de métodos científicos en la solución de sus problemas de producción. Este último fue realmente el determinante, dado que la empresa no quería correr riesgos que afectaran sus costos de producción y que le ocasionaran "probablemente" mayores pérdidas a las actuales. Los resultados le demostraron a la empresa que es posible beneficiarse mucho más, si se extiende el proceso de investigación a las demás presentaciones. Sin lugar a dudas, al hacerlo, las ventajas económicas permitirán adquirir tecnología avanzada, con lo que seguramente el proceso será capaz.

Investigaciones futuras podrían incluir la aplicación del método Taguchi, lo mismo que el uso de técnicas más avanzadas, como la metodología de superficies de respuesta para encontrar los niveles óptimos que aseguren la obtención del valor nominal especificado (Khuri y Cornell, 1991). Se sugiere la aplicación del Método Taguchi debido a que en esta técnica es posible incluir en el experimento factores de ruido con un mínimo de experimentación. Así pues, el objetivo en este método es lograr diseños robustos. Por otro lado, se encuentra la aplicación de la Metodología de Superficie de Respuesta la cual permitirá inspeccionar de manera visual y analítica el comportamiento de la variable de respuesta para cierta zona de los niveles de los factores de interés, y evaluar su sensibilidad. Para este caso el objetivo de aplicarla sería determinar la combinación de niveles de factores que proporcionaran una condición operativa óptima.

\section{AGRADECIMIENTOS}

A los Directores, Jefes, Gerentes, Supervisores y Operarios de la empresa por su infinita colaboración y disponibilidad para la realización del presente trabajo. 


\section{REFERENCIAS}

Albers Willem y C.M. Wilbert Kallenberg; Self-adapting control charts. Statistica Neerlandica: 60(3), 292-308 (2006).

Bisgaard Soren; Must a process be in statistical control before conducting designed experiments? Quality Engineering: 20, 143-150 (2008).

Bulba Edivaldo A., Linda Lee Ho; Capability index for relational functions: inferential Procedures. Quality Engineering. 18, 69-77 (2006).

Denniston Bill; Capability indices and Conformance to Specifications: the motivation for using $C_{p m}$, Quality Engineering: 18, 79-88 (2006).

Evans James R. y Lindsay William M.; "Administración y Control de la Calidad”. Sexta edición.516524. Editorial Thomson, D.F., México (2005).

Gijo E.V., Improving process capability of manufacturing process by application of statistical techniques. Quality Engineering: 17, 309-315 (2005).

Gutiérrez Pulido H. y R. De la Vara Salazar; "Control estadístico de calidad y seis sigma". 120130,328-364. Mc Graw-Hill. Guanajuato, México (2005).

Han C.-P.; Effect of testing normality on estimating process capability indices. Quality Engineering: 18, 391-395 (2006).

Hung-Chin Lin y Ji Shenn-Gwo; Practical Implementation of the capability index $C_{p k}$ based on the control chart data. Quality Engineering: 17, 371-390 (2005).

Khuri A. y J.A. Cornell; "Response Surfaces, Designs and Analyses”. Dekker, Dallas, Texas (1991).

Koning Alex J.; Model-based control charts in phase 1 statistical process control. Statistica Neerlandica: 60(3), 327-338 (2006).

Kotz S. y N. Johnson; Process Capability indices-a review, 1992-2000. American Society of Quality: 34(1), Enero , 2002.

Lloyd S. N. Is your process being adjusted too frequently? American Society of Quality: 35(1), Enero 2003.

Mathews Paul, "Design of Experiments with MINITAB". ASQ Quality Press, Milwaukee, Wisconsin (2005).

Montgomery. C.; "Introductions of Statistical quality control", John Wiley \&Sons, New York, (2001).

Peña Sánchez de Rivera Daniel y Albert Prat Bartés; "Cómo controlar la calidad", Editorial Madrid: Instituto de la Pequeña y Mediana Empresa Industrial, España (1986).

Polansky Alan M.; Permutation methods for comparing process capabilities. Journal of Quality Technology: 38(2), Junio (2006).

Shore Haim; Control charts for the queue length in a G/G/S system. IIE Transactions: 38, 1117-1130 (2006).

Tsai Tzong-Ru; Skew normal distribution and the design of control charts for averages. International Journal of Reliability, Quality and Safety Engineering: 14(1), 49-63 (2007).

Woodall William H.; The use of control charts in health-care and public-health surveillance. Journal of Quality Technology: 38(2), Abril (2006). 